\title{
Atherosclerosis is an inflammatory disease
}

\author{
Fabrizio Montecucco • François Mach
}

Published online: 17 April 2009

(C) Springer-Verlag 2009

During the last decades, emerging evidence has shown that atherosclerosis is characterised by a complex endocrine, paracrine and juxtacrine cross-talk between immune and vascular cells as well as several tissues and organs, including the liver, heart, kidney, adipose tissue, adrenal, pancreatic, pituitary and sex glands [1]. Thus, atherosclerosis has to be considered as a "systemic disease", characterised by arterial inflammatory lesions that mature and modify themselves with disease progression. Atherosclerotic plaques are the battlefield among an unbalanced immune response and lipid accumulation in the intima of arteries. Although the "first event" favouring early lipid deposition within the arterial sub-intimal space has not been identified, the infiltration of low-density lipoproteins (LDL) should be considered as the pivotal step in plaque formation [2]. Oxidative and enzymatic modifications of retained LDL increase the expression of adhesion molecules on endothelial cell surface membrane, thus favouring leukocyte recruitment from the blood stream [3]. Leukocytes recruitment within atherosclerotic plaques is also orchestrated by the chemokine binding to their receptors on cell membrane. This is a redundant system which regulates all inflammatory phases of atherogenesis in both humans and

\footnotetext{
F. Montecucco

Division of Cardiology, Foundation for Medical Researches, University Hospital,

Geneva, Switzerland

F. Mach $(\bowtie)$

Cardiology Division, Department of Medicine,

Geneva University Hospital, Foundation for Medical Researches,

64 Avenue Roseraie,

1211 Geneva, Switzerland

e-mail: francois.mach@unige.ch
}

animals [4]. Monocytes have been defined by Weber et al. as the "crucial force" to drive atherogenesis [3]. In vivo monocyte inflammatory functions and differentiation are influenced by the different expression of chemokine receptors, CD14 and CD16. Two different monocyte subsets have been identified. Inflammatory monocyte subset $\left(\mathrm{CXC}_{3} \mathrm{CR} 1{ }^{\text {low }} \mathrm{CCR} 2^{+} \mathrm{Ly} 6 \mathrm{C}^{\text {hi }} \mathrm{CD} 14^{+} \mathrm{CD} 16^{-}\right)$represents the main population recruited within the plaque from the blood stream and display both phagocytic and proteolytic activities. On the other hand, resident monocyte subset $\left(\mathrm{CXC}_{3} \mathrm{CR} 1{ }^{\text {hi }} \mathrm{CCR} 2{ }^{-} \mathrm{Ly} 6 \mathrm{C}^{\text {low }} \mathrm{CD} 14^{\text {low }} \mathrm{CD} 16^{+}\right)$is the main population involved in the initiation of tissue inflammation, angiogenesis and atheroprogression [3]. Modified LDL are uptaked by scavenger receptors of resident macrophages that differentiate into different inflammatory cell types, such as dendritic cells, foam cells, osteoclast- and osteoblast-like cells. These processes are modulated by several cytokines and growth factors (such as receptor activator of nuclear factor [NF]kappaB ligand [RANKL], osteoprotegerin [OPG], tumour necrosis factor [TNF]alpha, granulocyte macrophage-colony stimulating factor [GM-CSF], macrophage-colony-stimulating factor [M$\mathrm{CSF}]$ and interleukin [IL]-4). On the other hand, connexins, which mediate gap junction formation between adjacent cells (vascular and immune cells), have been recently shown to playing a crucial role in cell differentiation and growth. Three different connexins (Cx37, Cx40 and Cx43) are differently expressed in macrophage and endothelial cells during atherosclerotic plaque maturation [5]. At this step, the non-specific immune response, mainly characterised by local monocyte/macrophage-mediated inflammatory processes has started. In a second phase, resident inflammatory and vascular cells further produce chemokines for the recruitment of $\mathrm{T}$ lymphocytes within the plaque. This induces the deleterious vicious circle of the 
immune-mediated inflammatory response [6] $\mathrm{T}_{\mathrm{H}} 1$ lymphocytes, B cells, mast cells, neutrophils promote atherosclerotic processes. Conversely, $\mathrm{T}_{\mathrm{H}} 2$ and $\mathrm{T}_{\mathrm{reg}}$ lymphocytes rather reduce atherogenesis [3]. Both paracrine (chemokines and cytokines) and juxtacrine (mediated by adhesion molecules and connexins) communication pathways regulate leukocyte recruitment and intra-plaque activation $[3,5]$. An altered balance between $\mathrm{T}_{\mathrm{H}} 1$ cytokines (interferon [IFN]-gamma, IL-12, IL-15, IL-18 and TNF-alpha), antiinflammatory cytokines (TGF-beta and IL-10) and chemokines (mainly CCL2, CCL3, CCL5) increase immune cell functions and recruitment in atherosclerotic plaques. Adhesion molecules on both leukocytes (integrins CD11a, CD11b, CD11c, CD18 and selectins) and endothelial cells (ICAMs, VCAMs, PECAMs and JAMs) mediate cell rolling and adhesion to the vessel wall [7]. Local inflammation triggers "systemic" organs to release soluble inflammatory mediators, such as C-reactive protein (CRP), endocannabinoids, cytokines (IL-6, TNF-alpha, IFNgamma and adipocytokines) and hormones that further increase inflammation [8,9]. All these factors have been shown to directly induce both immune and vascular cell activation. Smooth muscle cells migrate into the intima and the arterial remodelling becomes irreversible which may cause arterial lumen stenosis with collagen and calcium deposition [9]. Once recruited into the intima, vascular and immune cells also release toxic mediators, such as proteases and oxidants that induce atherosclerotic plaque instability until its possible rupture. This dramatic event causes the exposure into the blood flow of intra-plaque pro-thrombotic material with the consequent formation of a thrombus. The sudden thrombotic occlusion of the artery at site of plaque disruption can stop the blood flow and induce critical ischemia in the heart, brain and peripheral tissues. The present issue of the Seminars in Immunopathology will focus on two different diseases, such as diabetes and sleep apnea syndrome (SAS) [10, 11], strongly associated to increased atherosclerosis and its dramatic complications. Finally, the present issue we will also focus on some promising therapies to reduce atherosclerosis. We will update new insights in vaccination approach and cannabinoid or statin treatments. Cannabinoid treatment in basic research, animal models and clinical studies reduced atherosclerosis and the risk of the acute ischemic complications [12-15]. The use of selective peripheral blockade of $\mathrm{CB}_{1}$ and/or the activation of $\mathrm{CB}_{2}$ cannabinoid receptors might represent a most promising therapy. However, the increase of major psychiatric adverse events that has been recently reported in clinical trials investigating the role of $\mathrm{CB}_{1}$ cannabinoid antagonist rimonabant in weight loss in obese patients, has strongly limited this approach $[15,16]$. Pleiotropic activities of statins represent another pivotal pathway to reduce atherosclerosis. In November 2008,
Ridker et al. have shown that rosuvastatin significantly reduced incidence of major cardiovascular events in a population of healthy subjects with elevated highsensitivity levels of CRP and without hyperlipidemia [17]. The efficacy of rosuvastatin in primary prevention independently of cholesterol lowering strongly suggest and support the use of these drugs to reduce CRPmediated pro-atherosclerotic risk. Together with vaccination approach, both cannabinoid and statin treatment will represent some very important approaches to reduce cardiovascular risk.

To summarise and introduce this issue, recent evidence confirmed that immune and inflammatory processes play a crucial role in all phases of atherosclerosis. Treatment with anti-inflammatory drugs represents a promising therapeutic approach to reduce atherosclerosis and its complications, such as myocardial infarction and stroke. In the next years, cardiovascular research will probably confirm that the selective regulation of immune response and inflammation will be the most important strategies to fight cardiovascular diseases.

Acknowledgements This research was funded by EU FP7, Grant number 201668, AtheroRemo and supported by a grant from the Swiss National Science Foundation to Dr. F. Mach (\# 320080105836).

\section{References}

1. Libby P (2007) Inflammatory mechanisms: the molecular basis of inflammation and disease. Nutr Rev 65:S140-146

2. Hansson GK (2005) Inflammation, atherosclerosis, and coronary artery disease. N Engl J Med 352:1685-1695

3. Weber C, Zernecke A, Libby P (2008) The multifaceted contributions of leukocyte subsets to atherosclerosis: lessons from mouse models. Nat Rev Immunol 8:802-815

4. Zernecke A, Shagdarsuren E, Weber C (2008) Chemokines in atherosclerosis: an update. Arterioscler Thromb Vasc Biol 28:1897-1908

5. Brisset AC, Isakson BE, Kwak BR (2009) Connexins in vascular physiology and pathology. Antioxid Redox Signal 11:267-282

6. Hansson GK, Libby P (2006) The immune response in atherosclerosis: a double-edged sword. Nat Rev Immunol 6:508-519

7. Braunersreuther V, Mach F (2006) Leukocyte recruitment in atherosclerosis: potential targets for therapeutic approaches? Cell Mol Life Sci 63:2079-2088

8. Mach F, Montecucco F, Steffens S (2008) Cannabinoid receptors in acute and chronic complications of atherosclerosis. $\mathrm{Br} \mathrm{J}$ Pharmacol 153:290-298

9. Hansson GK, Libby P, Schönbeck U et al (2002) Innate and adaptive immunity in the pathogenesis of atherosclerosis. Circ Res 91:281-291

10. Gu K, Cowie CC, Harris MI (1999) Diabetes and decline in heart disease mortality in US adults. JAMA 281:1291-1297

11. Masood A, Phillips B (2000) Sleep apnea. Curr Opin Pulm Med 6:479-484 
12. Montecucco F, Burger F, Mach F et al (2008) CB2 cannabinoid receptor agonist JWH-015 modulates human monocyte migration through defined intracellular signaling pathways. Am J Physiol Heart Circ Physiol 294:H1145-H1155

13. Steffens S, Veillard NR, Arnaud C et al (2005) Low dose oral cannabinoid therapy reduces progression of atherosclerosis in mice. Nature 434:782-786

14. Montecucco F, Lenglet S, Braunersreuther V et al (2009) $\mathrm{CB}_{2}$ cannabinoid receptor activation is cardioprotective in a mouse model of ischemia/reperfusion. J Mol Cell Cardiol . doi:10.1016/j. yjmcc.2008.12.014
15. Scheen AJ (2008) CB1 receptor blockade and its impact on cardiometabolic risk factors: overview of the RIO programme with rimonabant. J Neuroendocrinol 20(Suppl 1):139-146

16. Van Gaal L, Pi-Sunyer X, Després JP et al (2008) Efficacy and safety of rimonabant for improvement of multiple cardiometabolic risk factors in overweight/obese patients: pooled 1-year data from the Rimonabant in Obesity (RIO) program. Diabetes Care 31 (Suppl 2):S229-S240

17. Ridker PM, Danielson E, Fonseca FA et al (2008) Rosuvastatin to prevent vascular events in men and women with elevated Creactive protein. N Engl J Med 359:2195-2207 